\title{
Evaluación del control interno de compras en la fábrica de puros American Caribbean Cigars S.A
}

Brenda Lisseth Montoya Orozco ${ }^{1}$

1 Correo Electrónico: brelismont14@yahoo.es

\section{RESUMEN}

Este Artículo científico se escribió siguiendo líneas de investigación y de acción donde se plantea la evaluación el control interno de compras de American Caribbean Cigars, S.A. El tipo de estudio es descriptivo y de corte transversal porque se está evaluando un periodo siendo este el 2011.El enfoque es cualitativo con técnicas cuantitativas y cualitativas debido a que se realizan entrevistas las cuales son procesadas analíticamente de acuerdo a la valoración del funcionamiento de control interno, y encuestas procesadas cuantitativamente representadas en porcentajes según la muestra, se reforzó con investigación documental para confirmar la veracidad de la información brindada.

De forma específica el trabajo está elaborado siguiendo los componentes del modelo COSO I, los objetivos, y planteamiento del problema fueron abordados tomando la lógica de los componentes de control interno según el COSO. Según la problemática encontrada se corrobora que el control interno es deficiente analizando las grandes debilidades encontradas durante el proceso.

Por lo que se recomienda tomar medidas correctivas para solucionar las debilidades encontradas, en primer lugar se recomienda la elaboración de un manual de procedimientos de compras, un manual de funciones y un organigrama donde se establezcan las líneas de autoridad y responsabilidad, y que se realice una supervisión constante del proceso de compras.

Palabras claves: Control interno, proceso de compras, eficiencia, eficacia 


\title{
Evaluation of internal control shopping cigar factory American Caribbean Cigars S.A
}

Brenda Lisseth Montoya Orozco ${ }^{1}$

1 E-mail: brelismont14@yahoo.es

\begin{abstract}
This article was written following scientific lines of research and action where there is an evaluation of the internal control purchases of American Caribbean Cigars, S.A. This analysis is descriptive and cross-sectional because it is evaluating a period: 2011 year. The approach is qualitative and quantitative using techniques such as interviews that are analytically processed according to the evaluation of the internal control performance and surveys quantitatively processed showed in percentages as per the sample. The study was supported with a documentary research to confirm the accuracy of the information provided.
\end{abstract}

Specifically the thesis is prepared using the components of the COSO model I, the objectives and problem approach were raised following the logic of the internal control components stated by COSO. According to the problems observed it is confirmed that company's internal control is inadequate analyzing great weaknesses found during the process.

Therefore it's recommended to take measures addressed to solve the weaknesses found starting with the issue of a procurement procedure manual, a general operations manual and an organization chart that help to set up an appropriate route of authority and duties and to implement a permanent purchasing process control.

Keywords: Internal control, purchasing process, efficiency, effectiveness. 


\section{INTRODUCCIÓN}

Debido al crecimiento organizacional y las diferentes operaciones que las compras generan, este estudio centra su atención en la evaluación del control interno de compras de materiales en la fábrica de puros American Caribbean Cigars S.A, a fin de medir los efectos en la eficacia y eficiencia de las operaciones de compras, estimular el cumplimiento de políticas y leyes que rigen a la fábrica, y asegurar la validez de la información.

Pretende ser un aporte a todas aquellas empresas que no cuentan con un adecuado sistema de control interno de compras, en virtud de que las empresas día a día presentan mayor desarrollo y sólo mediante la obtención de su máxima eficiencia y eficacia operativa podrán mantenerse firmes y sólidas dentro de un mercado de competencia.

Para la realización de este trabajo se consultaron estudios vinculados con el tema de estudio, el problema no ha sido tratado con el mismo enfoque, pero si con temas relacionados. Evidentemente se constata que en Nicaragua no existen estudios concretos dentro de las fábricas de Puros, que evalúen el control interno de las compras como área específica.

El proceso de compras en la fábrica se hace de manera empírica, este proceso debería de estar fundamentado en políticas y normas que permitan el aseguramiento de la obtención de equipos, materiales, mercancías, a un precio correcto y de un proveedor adecuado, el cual se elija bajo las condiciones que este ofrezca ya sea de precio, de descuento, de formas de pago o de despacho. No cuentan con una persona encargada de la calidad de materia prima que se adquiere, poniendo en riesgo la calidad del producto que se ofrece.

Partiendo de la problemática existente en las condiciones operativas de la fábrica, se pretende evaluar el control interno de compras, con la finalidad de mejorar dicho proceso, y ajustar las necesidades de la fábrica, verificando de manera eficiente y que permita evidenciar el buen manejo de las operaciones que se realizan en el proceso de las compras, y la relación directa con otras áreas.

\section{MATERIALES Y MÉTODOS}

Se evaluó el control interno que está implementando la fábrica American Caribbean Cigars, S.A en el proceso de compras de materiales, mediante el tipo de estudio descriptivo, el cual consiste en describir un fenómeno sometiéndolo a una evaluación e identificando las debilidades encontradas para recomendar su mejora.

Este tipo de estudio se vale de técnicas convenientes para investigaciones in situ por lo que se utilizaron técnicas de entrevistas, encuestas y verificación documental, con ayuda de teorías expuestas en el marco teórico y de investigaciones realizadas por otros autores en campos de estudio similar, al igual que entrevistas realizadas a expertos en el tema.

Se realizaron encuestas y entrevistas individuales para cada uno de los integrantes de la muestra, esto para obtener una respuesta veraz y un punto de vista de cada uno de los que integran el proceso de compras en la Fábrica.

Método de investigación

El diseño de investigación que se utilizó el no experimental, de corte transversal porque se evaluó solo un periodo establecido el año 2011. Se estableció un enfoque cuali-cuantitativo, debido que se estableció una muestra reducida de investigación, y se abordan tanto técnicas cualitativas como los análisis en las entrevistas como también cuantitativas en las encuestas.

El estudio a utilizar para esta investigación fue de tipo inductivo ya que se parte de hechos particulares ocurridos en el proceso de compras de la fábrica 
American caribbean cigars, donde se enmarca el problema de investigación, para llegar a conclusiones. El diseño de investigación que se utilizó el no experimental, de corte transversal porque se evaluó solo un periodo establecido el año 2011.

Se estableció un enfoque cuali-cuantitativo, debido que se estableció una muestra reducida de investigación, y se abordan tanto técnicas cualitativas como los análisis en las entrevistas como también cuantitativas en las encuestas.

El estudio a utilizar para esta investigación fue de tipo inductivo ya que parte de hechos particulares ocurridos en el proceso de compras de la fábrica American caribbean cigars, donde se enmarca el problema de investigación, para llegar a conclusiones.

Inductivo: Según Bernal (2006):"establece que este método se utiliza para obtener conclusiones que parten de hechos particulares aceptados como válidos, para llegará conclusiones, cuya aplicación será de carácter general. Este método se inicia con un estudio individual de los hechos y se formulan conclusiones universales que se postulan con leyes, principios o fundamentos de una teoría" (p.56)

Se define para esta investigación la población a la fábrica de puros American Caribbean cigars, S.A. La Muestra fue el área de compras, al cual se dirigió el estudio específico y se involucraron a las siguientes personas: gerente general, administrador, responsable de recursos humanos, comprador, contador y bodeguero. Para un total de seis personas que están involucrados en el proceso de compras.

\section{RESULTADOS Y DISCUCIÓN}

Describiendo brevemente los resultados obtenidos se mencionan los más importantes siguiendo la lógica de los cinco componentes del marco COSO que se utilizó para la evaluación.
En lo que respecta al ambiente de control se encuentra que la segregación de funciones no es adecuada según información recabada. El administrador realiza las compras, y también se está desempeñando como contador provocando que las operaciones no correspondan a la eficiencia y eficacia esperada. Según información obtenida no cuenta con una estructura organizativa formal, donde se establezcan las líneas de autoridades y de responsabilidades por lo que en general el ambiente de control se percibe que no es el adecuado para un control efectivo y eficiente.

En cuanto a la evaluación de riesgos, se constata según revisión documental que no hay un plan contingente ante riesgos que puedan surgir en las diferentes actividades del proceso de compras, Por lo que no cuentan con las medidas correctivas para adoptarlas ante estos posibles riesgos.

Según información se obtiene que no cuente con pólizas de seguros para proteger inventarios, contra riesgos y siniestros, provocando con esto un inadecuado sistema de seguridad y lógica ante previsión de riesgos, no se cumple con la seguridad física de bienes y materiales.

En lo relativo a actividades de control las deficiencias más significativas se dan en la separación de tareas y responsabilidades, no existe control, ni seguridad razonable de la información debido a que una persona realiza las actividades más importantes del proceso de compras, el administrador que compra los materiales también registra la transacción, además aprueba y en algunos casos autoriza, y aun así lleva el control de los materiales inventariados, aumentando de manera significativa las probabilidades de fraude, posicionando a una persona como juez y parte, debido a que el maneja el control interno y también realiza las actividades. 
Se constata con una política que restrinja el acceso a recursos y activos, las actividades no son controladas por supervisión interna por lo que los riesgos en el proceso son inminentes.

En cuanto a supervisión y monitoreo no se realizan auditorias y tampoco hay supervisión interna de los procesos de compra por lo que se promueve de alguna manera a la ilegalidad de procedimientos dentro de la fábrica., provocando posibilidad de riesgos y deficiencia en las operaciones.

No han establecido políticas de compras concretas lo que provoca que la fábrica no cuente con una seguridad y confiabilidad razonable, esto provoca que no exista control establecido en las actividades que se realicen en el proceso, debido a que no tienen una legalidad establecida que sirva como guía para realizar de manera eficiente y efectiva las actividades, se han seguido como políticas los requisitos establecidos por la ley 382 ley de admisión temporal de mercancía, sin fijarse en los objetivos estratégicos propios para el desarrollo de la fábrica que conlleve al crecimiento esperado.

\section{RECOMENDACIONES}

Atendiendo el análisis de resultados y las conclusiones se recomendó:

- La gerencia en conjunto con la administración deberá establecer políticas de compras que especifiquen los procedimientos operativos y las prácticas de negociaciones con los proveedores, la selección y otros aspectos similares que permitan regular las actividades a realizar.

- La gerencia deberá contratar a un contador para el registro oportuno de las transacciones, atendiendo a la problemática de que una persona este autorizando, registrando y pagando las compras de materiales.

- La gerencia deberá girar instrucciones para que las compras sean autorizadas por el gerente, sin importar el monto y el tipo de materiales que se compren.

- La administración en conjunto con la Gerencia deberá de diseñar un control sobre los procedimientos de compras, que este separado del departamento que las recibe, de su contabilización, y de su pago.

- El Gerente deberá supervisar constantemente el proceso de compras en todas sus actividades, así se evitan errores, omisiones, y actos ilícitos que puedan surgir.

- La administración deberá invertir en mantenimiento y mejoras en los sistemas de información, y en el pago de pólizas de seguros contra robo y siniestros.

- El administrador deberá orientar a los usuarios de sistemas de información que respalden la información con dispositivos externos de almacenamiento de datos, en vista a incendios y siniestros naturales que puedan surgir.

- La gerencia y administración deberá realizar una estructuración formal de la fábrica, en cuanto a la elaboración de un organigrama adecuado para el tipo de empresa, elaborar manuales de procedimientos, manuales de funciones.

- El administrador deberá especificar las funciones de cada empleado de acuerdo al puesto que desempeñe.

- La Administración deberá elaborar misión, visión, y objetivos estratégicos será de importancia para establecerse claramente y hacerles saber a los trabajadores quienes son y a donde quieren llegar, de manera que se trabaje en función de alcanzarlo.

\section{BIBLIOGRAFÍA}

Bernal, Cesar Augusto (2006). Metodología de la investigación. México: segunda Edición Edición: Pearson Educación de México.

Coopers y Lybrand (1997). Los nuevos conceptos del control interno (Informe Coso) , España, Ediciones 
Díaz de Santos S.A.

Díaz de Santos (1995). Compras e Inventarios. Ediciones : Díaz de santos, Madrid España.

Mantilla B, Samuel Alberto (2003). Control Interno Informe Coso. Editorial ECOE EDICIONES. Cuarta Edición. Colombia.

Montoya Palacios, Alberto (2002). Administración de compras. Bogotá: Editorial Norma.

Perdomo Moreno, Abraham (2004). Fundamentos de control Interno, Novena Edición, Editorial Thomson.

Stephen, R y Coulter, M. (2005). Administración. Edición: Marisa de Anta: octava edición, México. 